\title{
Thyroid Ultrasound Findings and Its Associated Parameters in Saudi People
}

Mogahed MM*, El-Awady $\mathrm{MA}^{2}$ and Samih $\mathrm{TA}^{3}$ ${ }^{1}$ Department of Internal Medicine, Faculty of Medicine, Benha University, Egypt

${ }^{2}$ Department of Public Health, Faculty of Medicine, Benha University, Egypt

${ }^{3}$ Department of Radiology, Faculty of Medicine, Benha University, Egypt

*Corresponding author: Mysara Mohamad Mogahed, Department of Internal Medicine, Faculty of Medicine, Benha University, Egypt

Received: March 15, 2021; Accepted: April 09, 2021; Published: April 16, 2021

\section{Abstract}

Background: Thyroid abnormalities are the most common endocrine abnormalities in Saudi Arabia (KSA). Ultrasonography (USG) is the commonest method of thyroid gland imaging. Thyroid lesions prevalence depends on factors such as sex, age, iodine intake, and other metabolic parameters.

Objectives: Investigating different thyroid lesions and their associated clinical parameters.

Subjects and Methods: This is a cross-sectional study of adult residents who visited Internal Medicine Clinic in Riyadh, KSA. 206 participants were subjected to thyroid USG, weight and height measurement, laboratory investigations including FBG, HbA1C, ALT, AST, creatinine, uric acid, lipid profile, thyroid stimulating hormone (TSH), and FT4.

Results: Thyroid nodules were found in $27.2 \%, 30.1 \%$ had thyroiditis, and $6.8 \%$ had pure cysts. There was a significant prevalence of thyroid ultrasound findings within different age groups, $(P=0.002)$. The highest $B M I$ was found in subjects with nodular size $\leq 1 \mathrm{~cm}$. Subjects with thyroid nodules had higher level of FBG, HbA1c, ALT, AST, uric acid, total cholesterol, HDL-C, LDL-C and TSH. The most frequent thyroid status was euthyroid, followed by subclinical hypothyroidism which had higher frequency in subjects with nodular size $\leq 1 \mathrm{~cm}$ $(30.8 \%)$.

Conclusion: Imaging, particularly USG, plays an important role in classifying thyroid gland abnormalities. We found that thyroid lesions, including nodules, pure cysts, and thyroiditis, were non-significantly positively associated with disturbed metabolic profile such as high FBS, HBA1C, ALT, AST, total cholesterol, and LDL levels. Our results for biochemical parameters do not allow inferences on whether they are independent factors associated with thyroid lesions or not.

Keywords: Thyroid lesion; Ultrasonography; Metabolic parameters

\section{Introduction}

Thyroid is an endocrine gland, and its involvement has diverse functions and concerns; the major concern with thyroid lesion is the possibility of malignancy, and other minor concerns are related to cosmetics and appearances. Thyroid lesions are not only a sign of expression of a single disease but also a clinical manifestation of a wide range of different diseases [1].

Thyroid gland disorders are the most common endocrine abnormalities in KSA and in the whole Middle East region. Thyroid lesion can be classified into congenital, inflammatory, endocrinal, and neoplastic (benign and malignant) [2].

Ultrasonography (USG) is extensively reported to be the most common and useful method of imaging thyroid gland and its abnormalities [3].

Thyroid enlargement, like thyroid nodules, is detected more objectively by USG than physical examination. Nodules with suspicious ultrasound features should be considered for biopsy [4] An abnormal USG pattern of the thyroid, characterized by diffuse low echogenicity, was reported in autoimmune thyroid diseases, and its potential value shows in the diagnosis of Hashimoto's thyroiditis [5].

Investigating thyroid disorders is important to evaluate malignancy risk [6]. As the incidence of thyroid cancer has tripled in the last 30 years, proper investigation of thyroid disorders and associated factors is fundamental to define a better diagnostic approach [7].

The contribution of the increasing detection rate is a widely accepted explanation for the increasing prevalence of thyroid nodules. It is well established that thyroid nodule prevalence depends on factors such as the detection method, sex, age, iodine intake, and radiation exposure [8]. Other risk factors including metabolic parameters have received attention recently. Previous studies have shown that insulin resistance was associated with increased thyroid nodule prevalence in iodine-deficient and iodine-sufficient areas $[9,10]$. A recent study also reported that hypertension, prediabetes, and diabetes were independent risk factors for thyroid nodules [11]. Body size parameters such as height, weight, and (BMI) were reported to be associated with the presence of thyroid nodules in women [12].
Annals Thyroid Res - Volume 7 Issue 2 - 2021

Submit your Manuscript | www.austinpublishing group.com Mogahed et al. (C) All rights are reserved 
The aim of this cross-sectional study was to determine thyroid ultrasound findings in Saudi people, to investigate different gland lesions and their associated clinical parameters.

\section{Subjects and Methods \\ Study design}

This is a cross-sectional study of adult residents in Riyadh, KSA, who visited the Internal Medicine Clinic in a polyclinic medical center.

\section{Data collection}

A total of 206 health checkups were performed from January 2020 to December 2020. Thyroid USG was performed to all. We did not exclude the subjects with other diseases, including diabetes, hypertension, dyslipidemia, and thyroid dysfunction as well as the subjects using any kind of medication, including thyroid hormone or antithyroid drugs. Informed consent was obtained.

\section{Anthropometric parameters}

We measured the height and weight of subjects in light clothing and without shoes to the nearest $0.1 \mathrm{~cm}$ and $0.1 \mathrm{~kg}$, respectively. BMI was calculated as the ratio between the weight and the square of height, and it was expressed in $\mathrm{kg} / \mathrm{m}^{2}$.

\section{Biochemical and hormonal parameters}

Plasma glucose concentration was measured in the 12-hour fasting state using the glucose oxidase and a modified Trinder color reaction, catalyzed by the peroxidase enzyme method. Glycosylated hemoglobin (HbAlc) level was measured using the well-established method of boronate affinity. The serum concentrations of Aspartate Aminotransferase (AST), Alanine Aminotransferase (ALT), creatinine, uric acid, total cholesterol, triglycerides, Low-Density Lipoprotein Cholesterol (LDL-C), and High-Density Lipoprotein Cholesterol (HDL-C) were measured by Konelab kits using Indiko fully automated analyzer. The concentrations of serum free Thyroxine (T4) and TSH were measured using the chemiluminescence assay (Roche Diagnostics, Cobas 6000 analyzer e601 module, Germany). The normal range according to the manufacturer for TSH and free T4 was 0.4 to $5 \mathrm{mIU} / \mathrm{L}$ and 10.6 to $19.4 \mathrm{pmol} / \mathrm{L}$, respectively.

\section{Classification of thyroid function status}

The subjects were divided in five categories based on their thyroid function status:

- Overt hypothyroid state was defined as a TSH level $>5 \mathrm{mIU} / \mathrm{L}$ with a free $\mathrm{T} 4$ level $<10.6 \mathrm{pmol} / \mathrm{L}$.

- Subclinical hypothyroid state was defined as a TSH level $>5 \mathrm{mIU} / \mathrm{L}$ with a normal free T4 level (10.6 to $19.4 \mathrm{pmol} / \mathrm{L}$ ).

- $\quad$ Euthyroid state was defined as a normal TSH level (0.40 to $5 \mathrm{mIU} / \mathrm{L})$.

- Subclinical thyrotoxic state was defined as a TSH level $<0.40 \mathrm{mIU} / \mathrm{L}$ with a normal free T4 level

- Overt thyrotoxic state was defined as a TSH level $<0.40 \mathrm{mIU} / \mathrm{L}$ with a free T4 level $>19.6 \mathrm{pmol} / \mathrm{L}$.

We did not consider the use of thyroid hormone and antithyroid drugs in defining thyroid function status.

\section{Thyroid ultrasound and the classification of thyroid nodules}

All ultrasonographic examinations of the thyroid were performed for each participant by a radiologist using high-resolution US equipped with 7.5-12 or 8-15 MHz linear transducers. Thyroid nodules were defined as one or more discrete lesions that were within the thyroid gland but were radiologically distinct from the surrounding thyroid parenchyma. The length, width, and depth of each nodule were measured at their longest aspect on the US images, which were saved as digital images. All US reports were also saved as digital text. We extracted data of the presence and size of nodules from the free text of the thyroid US reports. According to the lesion's content, a lesion in which the cystic portion comprised $\geq 95 \%$ of the volume was defined as a pure cyst, and a lesion in which the solid portion comprised $6 \%$ to $100 \%$ of the volume was defined as a nodule. If the extracted data harbored the keywords "cyst" and "nodule" in one subject, this was classified as a nodule. In the subjects with multiple nodules, the size of the largest thyroid nodule was used in the analyses.

\section{Statistical analysis}

SPSS Software (version 26.0 for Windows) was used. Qualitative data were summarized as frequency and percentage, while quantitative data as Mean \pm Standard Deviation (SD), median, and Interquartile Range (IQR) after establishing nonnormality of some variables by K-S test (One-Sample Kolmogorov-Smirnov Test) of normality. The Student $t$-test and Wilcoxon test were applied for the comparison of quantitative variables between two groups for both parametric and nonparametric data. Comparisons between the different study groups were carried out using the chi-square test and the Fisher exact test to compare proportions as appropriate. Statistical significance was accepted at $\mathrm{P}<0.05$.

\section{Results}

\section{Basic information of the studied population}

A total of 206 subjects were enrolled in the study, with 30 men and 176 women, with a median age of 33 years, IQR of $28-41$ years, and mean BMI of $29.84 \pm 6.66 \mathrm{~kg} / \mathrm{m}^{2}$. Thyroid nodules were found in 56 ( 26 with nodular size $\leq 1 \mathrm{~cm}$ and 30 with nodular size $>1 \mathrm{~cm}$ ) subjects (27.2\%), thyroiditis was found in $62(30.1 \%)$ subjects, and 14 subjects (6.8\%) had only pure cysts. Other ultrasonographic findings include atrophied thyroid (4) and thyroidectomy (4) with percentage of $1.9 \%$ for each, while normal thyroid was found in 66 (32.1\%). Among the 30 men, 6 (20\%) and 8 (26.7\%) subjects were diagnosed with thyroid nodules and thyroiditis, respectively, while no subject (0\%) was as diagnosed with pure cyst. Among the 176 women, 50 (28.4\%) and 54 $(30.7 \%)$ subjects were diagnosed with thyroid nodules and thyroiditis, respectively, while $14(8 \%)$ subjects were diagnosed with pure cyst. According to age stratification, there was a significant difference in the prevalence of thyroid ultrasound findings in different age groups, with pure cyst being associated with older age group $(\mathrm{KW}=20.29$, $\mathrm{P}=0.002$ ).

\section{Comparison of Body Mass Index in Different Thyroid US Findings}

After exclusion of subjects with atrophied thyroid and those with thyroidectomy, the highest BMI was found in subjects with nodular size $\leq 1 \mathrm{~cm}(32.4 \pm 10.6)$; however, there was no significant statistical 
difference in BMI in different studied subjects as regards their thyroid US findings.

\section{Comparison of biochemical and metabolic parameters in different thyroid us findings}

After excluding 8 subjects who had thyroidectomy and congenital thyroid atrophy, we found that subjects with thyroid nodules had higher level of fasting glucose, HbA1c, ALT, AST, uric acid, total cholesterol, LDL-C, and TSH in comparison with other groups, while the concentration of creatinine and FT4 was higher in thyroiditis and TGS was highest in the group with pure cyst.

\section{Association between thyroid function status and different thyroid us findings}

The most frequent thyroid status was euthyroid state in different ultrasound thyroid findings, followed by subclinical hypothyroidism which had higher frequency in subjects with nodular size $\leq 1 \mathrm{~cm}$ (30.8\%). The lowest recorded frequency was overt hyperthyroidism which was observed in only 4 patients with thyroiditis. This was highly statistically significant with a p value $<0.001$.

\section{Discussion}

Thyroid disorders are among the commonest endocrine disorders reported worldwide. Most of the people at risk of developing thyroid disorders are from developing countries, including KSA. The prevalence of thyroid disorders differs according to sex, age, lifestyle, dietary changes, diabetes, hypertension, and metabolic diseases such as hyperlipidemia, fatty liver, hyperuricemia, and obesity [13]. Classifying thyroid lesions is challenging; nonetheless, using ultrasonography may allow for accurate diagnosis, differentiation, and management of thyroid lesions [14]. The aim of this study was to determine thyroid ultrasound findings in Saudi people, to investigate different gland lesions and their associated clinical parameters.

In our current study, there was a significant relation between age and different thyroid lesions as follows: the median age of subjects diagnosed with thyroiditis was 30 years; solitary nodules occurred during the age of 30-40, which was lower than the age distribution of individuals with solitary thyroid nodules in the study of Bing Zou et al. [15], who studied the prevalence of thyroid nodules in Chinese and found that solitary thyroid nodules were most prevalent in the age of 70-89, and also lower than the age distribution of individuals with solitary thyroid nodules in the study of Moon JH, et al. [16], who studied the prevalence of thyroid nodules in Koreans and found that solitary thyroid nodules were most prevalent in a mean age of 52.7 . In the study conducted by $\mathrm{Tu}$ w, et al. [17] in China, they reported that nodular lesions were more frequent in population $\geq 60$ years, either diabetic or nondiabetic. Our finding was higher than those in the study of Kishan and Prasad [18] who reported that the highest incidence of solitary nodule was recorded during the third decade of life in Indians. Pure cyst occurred at a median age of 42, which was in agreement with Saeed et al. [19] who explored the pattern of thyroid lesions in western region of Saudi Arabia and found that colloid nodules (cysts) were more frequent in the age of 41-50. A number of epidemiological investigations worldwide show that the prevalence of thyroid nodules increases with age $[20,21]$. The mechanism may be that, with the increase of age, the thyroid will undergo degenerative changes, leading to diffuse compensatory hyperplasia of the thyroid and eventually the nodules [22]. This was not consistent with our data, maybe due to the younger age of our studied group as the median age of our participants was 33 .

In this cross-sectional study, the prevalence of thyroid nodules in health checkup participants in Saudi Arabia was 27.2\%, which is nearly similar to the study of Saeed et al. [19], who found that solid nodules represent $28.3 \%$ of their study population, and also the study of Siqueira et al. [23], who studied the factors associated with the occurrence of thyroid nodules in severely obese patients in Brazil and reported that the occurrence of nodules was $29.9 \%$ in their obese group. It is also consistent with the findings of Dauksiene et al. [24], who studied the factors associated with thyroid nodules in Lithuanian subjects and noted that thyroid nodules represent $31.2 \%$. Other countries have nearly similar prevalence of thyroid nodules (China $32.4 \%$ and Korea 34.2\%) [15,16]. The patterns of cystic thyroid lesions observed in this study were not comparable with other available literature; cystic thyroid lesion was observed in $6.8 \%$ of our participants. Moon JH, et al. [16] have reported that the prevalence of thyroid cyst was $12.5 \%$. A concordance rate of $8.9 \%$ was also noted by Almohiy et al. [25], who evaluated the utility of ultrasound examination in assessing thyroid abnormalities in Aseer region of KSA. In our study, out of 206 cases in total, $30.1 \%$ cases were diagnosed with thyroiditis, which is much higher than the prevalence of thyroiditis in the study of Saeed et al. [19] and Almohiy et al. [25], who reported $11.3 \%$ and $0.4 \%$ cases of thyroiditis, respectively. Many centers have adopted the method of ultrasound for the diagnosis of various thyroid problems, and this will help in detecting the thyroid lesions especially tumors in an early stage. Improvement in the detection of thyroid lesions became easy by extensive use of USG of thyroid; the USG detects lesions in up to $67 \%$ of the population [26]. Thyroid lesions are common and usually benign. The analysis of the frequencies of thyroid disorders by ultrasound imaging would assist in initiating appropriate and optimal long-term thyroid health management for the residents of Riyadh, KSA.

The results of this study show that gender is a non-significant risk factor for thyroid lesions, and its prevalence in women is higher than that in men, which is consistent with the previous reports [19,23]. But other studies [15-17] show that gender is an independent risk factor for occurrence of thyroid lesions. The high incidence of thyroid lesions in women is associated with increased demand for thyroid hormones during pregnancy, breastfeeding, and menstruating; estrogen can also affect the development of thyroid nodules [27].

We also observed a non-significant association between different thyroid lesions and BMI as thyroid nodules were associated with the highest BMI, while lowest BMI was present in normal thyroid USG, which was in accordance with earlier studies $[15,16,24,25]$ that reported an independent association between the presence of thyroid nodules and a higher BMI.

In this study, FBS, HBA1C, ALT, AST, total cholesterol, and LDL levels were higher and HDL levels were lower in patients with thyroid nodules in relation to other thyroid lesions, which is also compatible with previous studies $[15,16,24,25]$. However, whether these metabolic alterations independently favor the occurrence of thyroid nodules remains unclear [28,29]. 
In our data, TSH level was higher in the subjects with thyroid nodules compared with those with other lesions, and the nodular group had a higher frequency of overt/subclinical hypothyroid state and no recorded cases of overt thyrotoxic state.

One of the hypotheses that explain the relation between thyroid nodules and metabolic disturbances is a TSH dependent mechanism including leptin signaling. Leptin increases the secretion of thyrotropin-releasing hormone, which results in an increase of TSH secretion [30]. Serum leptin increases in proportion to increased fat mass [31]. Thus, it has been postulated that leptin signaling increases TSH secretion, which results in thyroid volume expansion and nodule formation. However, in our data, metabolic parameters and different thyroid status were non-significantly related to thyroid lesions, which were also observed by other studies [23,24]. And in contrast to our finding was the study of Moon $\mathrm{JH}$, et al. [16], who found that TSH level, was lower in the subjects with thyroid nodules compared with those without nodules, and the nodular group had a higher frequency of overt/subclinical thyrotoxic state and lower frequency of overt/subclinical hypothyroid state. Thus, a non-TSH dependent mechanism might be involved in the association between different thyroid lesions and metabolic disturbances.

The strength of the present study includes the use of the data from randomly selected participants. A further strength is extensive phenotypic characterization of participants, which includes an elaborate set of anthropometric measurements and metabolic factors, as well as a complete thyroid status assessment with measurements of TSH and FT4. The narrow age range of subjects eliminates the potential effect of age that may influence the observed associations among variables.

\section{Study Limitation}

There are several limitations to our study. First, the study had a relatively small sample size, which may lead to over- or underestimation of the study findings. Second, the study could not evaluate autoimmunity of the thyroid gland or iodine intake, both of which strongly influence the ultrasound imaging of the thyroid gland. Third, no FNA or cytological examination of thyroid nodules was done.

\section{Conclusion}

In conclusion, we conducted this study to investigate different thyroid ultrasound findings and their associated clinical parameters, in Saudi people. We found that ultrasonographic examination is a very valuable diagnostic tool in the evaluation of thyroid disorders. Imaging, particularly USG, plays an important role classifying various thyroid gland abnormalities. The high prevalence of thyroid lesions detected by thyroid USG at health checkups suggests that increased detection of thyroid lesions has resulted in an increasing prevalence in the general population. We document that thyroid lesions were significantly affected by age. We also found that thyroid lesions including nodules, pure cyst, and thyroiditis were non-significantly positively associated with disturbed metabolic profile such as high FBS, HBA1C, ALT, AST, total cholesterol, and LDL levels. Our results for biochemical parameters do not allow inferences on whether they are independent factors associated with thyroid lesions or not. Considering the controversy of the subject and the innovative contribution of this study, we stimulate further longitudinal investigations to clarify this question. Although our study is just a preliminary step towards identifying thyroid gland abnormalities, it would nevertheless assist in the long-term management of the thyroid health of residents of KSA.

\section{Acknowledgement}

The authors would like to express their special thanks all participants of the study and to all those who helped us during the whole course of the study. We acknowledge with deep sense of gratitude, the encouragement and inspiration we received from colleagues and the members of staff in the department of internal medicine, Benha University.

\section{References}

1. Rallison ML, Dobyns BM, Meikle AW, Bishop M, Lyon JL, Stevens W. "Natural history of thyroid abnormalities: prevalence, incidence, and regression of thyroid diseases in adolescents and young adults". Am J Med. 1991; 91: 363370 .

2. Al Shahrani AS, El-Metwally A, Al-Surimi K, Bin SalihS, Saleh Y, Al-Shehri A et al. "The epidemiology of thyroid diseases in the Arab world: A systematic review". J Public Health Epidemiol. 2016; 8:17-26.

3. Yunus $M$ \& Ahmed Z. "Significance of ultrasound feature $s$ in predicting malignant solid thyroid nodules: Need for fine -needle aspiration". J Pak Med Assoc. 2010; 60: 848-853.

4. Kovacevic DO \& Skurla MS. "Sonographic diagnosis of thyroid nodules: correlation with the results of sonographically guided fine -needle aspiration biopsy". J Clin Ultrasound. 2007; 35: 63-67.

5. Anderson L, Middleton W D, Teefey SA, e t al. "Hashimoto thyroiditis: Part 1, Sonographic analysis of the nodular form of Hashimoto thyroiditis". AJR. 2010; 195: 208-215.

6. Hegedus L. "Clinical practice. The thyroid nodule". N Engl J Med. 2004; 351: 1764-1771.

7. Davies L, Morris LG, Haymart M, et al. "American Association of Clinical Endocrinologists and American College of Endocrinology disease state clinical review: the increasing incidence of thyroid cancer". Endocr Pract. 2015; 21: 686-696.

8. Knudsen N, Laurberg P, Perrild H, Bulow I, Ovesen L, Jorgensen T. "Risk factors for goiter and thyroid nodules". Thyroid. 2002; 12: 879-888.

9. Rezzonico J, Rezzonico M, Pusiol E, Pitoia F, Niepomniszcze H. "Introducing the thyroid gland as another victim of the insulin resistance syndrome". Thyroid. 2008; 18: 461-464.

10. Ayturk S, Gursoy A, Kut A, Anil C, Nar A, Tutuncu NB. "Metabolic syndrome and its components are associated with increased thyroid volume and nodule prevalence in a mild-to-moderate iodine-deficient area". Eur $\mathrm{J}$ Endocrinol. 2009; 161: 599-605.

11. Guo $H$, Sun $M, H e ~ W$, et al. "The prevalence of thyroid nodules and its relationship with metabolic parameters in a Chinese community-based population aged over 40 years". Endocrine. 2014; 45: 230-235.

12. Kim JY, Jung EJ, Park ST, et al. "Body size and thyroid nodules in healthy Korean population". J Korean Surg Soc. 2012; 82: 13-17.

13. Statistics by Country for Thyroid disorders. 2016.

14. Al-Ghanimi IA, Al-Sharydah AM, Al-Mulhim S, Faisal S, Al-Abdulwahab A, Al-Aftan $\mathrm{M}$, et al. "Diagnostic accuracy of ultrasonography in classifying thyroid nodules compared with fine-needle aspiration". Saudi J Med Med Sci. 2019; 8: 25-31.

15. Zou B, Sun L, Wang X and Chen Z. "The Prevalence of Single and Multiple Thyroid Nodules and Its Association with Metabolic Diseases in Chinese: A Cross-Sectional Study". International Journal of Endocrinology. 2020; 2020: 5381012. 
16. Moon JH, Hyun MK, Lee JY, Shim JI, Kim TH, Choi HS, et al. "Prevalence of thyroid nodules and their associated clinical parameters: a large-scale, multicenter-based health checkup study". Korean J Intern Med. 2018; 33: 753-762.

17. Tu W, Zhang G, Yu S, Tang J and Yu J. "Observations on factors that influence thyroid nodules in diabetic and non-diabetic patients in the Zhejiang province of China". Int J Clin Exp Med. 2015; 8: 19332-19338.

18. Kishan AM. \& Prasad K. "Prevalence of solitary thyroid nodule and evaluation of the risk factors associated with occurrence of malignancy in a solitary nodule of thyroid". Int Surg J. 2018; 5: 2279-2285.

19. Saeed MI, Hassan AA, Buttc ME, Baniyaseen KA, Siddiquie MI, Bogarif NM, et al. "Pattern of Thyroid Lesions in Western Region of Saudi Arabia: A Retrospective Analysis and Literature Review". Journal of Clinical Medicine Research. 2018; 10: 106-116.

20. J Yin, C Wang, Q Shao, et al. "Relationship between the prevalence of thyroid nodules and metabolic syndrome in the iodine-adequate area of Hangzhou, China: a cross-sectional and cohort study". International Journal of Endocrinology. 2014; 2014: 675796.

21. $\mathrm{H}$ Guo, M Sun, W He, et al. "The prevalence of thyroid nodules and its relationship with metabolic parameters in a Chinese community-based population aged over 40 years". Endocrine. 2013; 45: 230-235.

22. N Kwong, M Medici, TE Angell, et al. "The influence of patient age on thyroid nodule formation, multinodularity, and thyroid cancer risk". The Journal of Clinical Endocrinology \& Metabolism. 2015; 100: 4434-4440.

23. Siqueira RA, Noll M, Rodrigues APS \& Silveira EA. "Factors Associated with the Occurrence of Thyroid Nodules in Severely Obese Patients: A CaseControl Study". Asian Pacific Journal of Cancer Prevention. 2019; 20: 693697.
24. Dauksiene D, Petkeviciene J, Klumbiene J, Verkauskiene R, Vainikonyte-K J, Seibokaite A, et al. "Factors Associated with the Prevalence of Thyroid Nodules and Goiter in Middle-Aged Euthyroid Subjects". International Journal of Endocrinology, 2017; 2017: 8401518.

25. Almohiy $H$, Adam M, Adam E \& M Al-Musa H." Evaluating the Utility of Ultrasound Examination in Assessing Thyroid Gland Abnormalities in Saudi Arabian Population". Pakistan Journal of Radiology. 2017; 27.

26. Ezzat S, Sarti DA, Cain DR, Braunstein GD. "Thyroid incidentalomas. Prevalence by palpation and ultrasonography". Arch Intern Med. 1994; 154 1838-1840.

27. K Wang, Y Yang, Y Wu, J Chen, D Zhang and C Liu. "The association of menstrual and reproductive factors with thyroid nodules in Chinese women older than 40 years of age". Endocrine. 2015; 48: 603-614.

28. Rezzonico JN, Rezzonico M, Pusiol E, Pitoia F, Niepomniszcze H. "Increased prevalence of insulin resistance in patients with differentiated thyroid carcinoma". Metab Syndr Relat Disord. 2009; 7: 375-380.

29. Sousa PA, Vaisman M, Carneiro JR, et al. "Prevalence of goiter and thyroid nodular disease in patients with class Illobesity". Arq Bras Endocrinol Metabol. 2013; 57: 120-125.

30. Zimmermann-Belsing T, Brabant G, Holst JJ, Feldt-Rasmussen U. "Circulating leptin and thyroid dysfunction. Eur J Endocrinol. 2003; 149: 257-271.

31. Schmidt MI, Duncan BB, Vigo A, et al. "Leptin and incident type 2 diabetes: risk or protection"? Diabetologia. 2006; 49: 2086-2096. 